\title{
Isotope effect of diffusion in a simple liquid
}

\author{
M. Kluge and H. R. Schober \\ Institut für Festkörperforschung, Forschungszentrum Jülich, D-52425 Jülich, Germany
}

(Received 27 October 1999)

\begin{abstract}
The diffusional isotope effect of a monatomic Lennard-Jones liquid is calculated by molecular-dynamics simulation. Mass differences of $10-40 \%$ were used. At equilibrium density, with decreasing temperature a strong reduction of the isotope effect is found that indicates a marked increase of the collectivity of motion. Changing the density at constant temperature, the same effect is seen that shows that the density change is the main driving force behind the reduction of the isotope effect.
\end{abstract}

PACS number(s): 66.10. $-\mathrm{x}$, 64.70.Pf, 82.20.Tr, 82.20.Wt

The atomic nature of transport in liquids has been the subject of numerous studies, e.g., [1-4]. Of particular interest is the possible change of mechanism upon cooling to melting point and beyond to the glass transition. In a monatomic liquid, the diffusion constant can be written

$$
D=D_{0} f(T, \rho)=D_{0}^{*} f(T, \rho) / \sqrt{m},
$$

where $T$ is the temperature, $\rho$ the atomic density, and $m$ is the mass of the diffusing particle. The temperature dependence of $D$ can be described over a large temperature interval down into the undercooled regime by a Vogel-FulcherTamman (VFT) law

$$
D=D_{0} \exp \left(\frac{E_{0}}{k_{\mathrm{B}}\left(T-T_{0}\right)}\right),
$$

where the phenomenological VFT temperature $T_{0}$ is well below the glass transition temperature $T_{\mathrm{g}}$. This law provides an empirical description of the sharp drop of $D$ upon undercooling. The inverse proportionality to the root of the mass is quite general, independent of the molecular aggregation, as long as only one species is present.

Already, in the simple case of different isotopes, considered here, the situation is more complicated. At low densities and high temperatures when diffusion is dominated by binary collisions, the kinetic approximation should hold and Eq. (1) should apply approximately for each component. Lowering the temperature or increasing the density effects of collective motion will gain importance. This cooperativity can be accounted for simply by replacing the particle mass in Eq. (1) by an effective mass for diffusion of isotope $\alpha$,

$$
m_{\mathrm{eff}}^{\alpha}=m^{\alpha}+\left(N_{D}-1\right) \bar{m},
$$

with $\bar{m}$ the average particle mass and $N_{D}$ the number of particles moving cooperatively. Equation (1) is then modified to

$$
D=F(T, \rho) / \sqrt{m_{\text {eff }}} .
$$

With this, one can define an isotope effect parameter $E$ [5],

$$
E_{\alpha \beta}=\frac{D_{\alpha} / D_{\beta}-1}{\sqrt{m^{\beta} / m^{\alpha}}-1} \approx \frac{1}{N_{D}} .
$$

The isotope effect can thus be used as a quantitative measure of collectivity. Due to the small differences in isotopic masses, it necessitates, however, very accurate measurements of the diffusion. Early measurements, therefore, often lead to conflicting evidence [1]. A substantial isotope effect was measured in molten $\mathrm{Li}$ [6].

Progress was made by simultaneously measuring the diffusion of the tracer atoms ${ }^{57} \mathrm{Co}$ and ${ }^{60} \mathrm{Co}$ [7]. Using this technique for diffusion of $\mathrm{Co}$ in amorphous $\mathrm{Co}_{76.7} \mathrm{Fe}_{2} \mathrm{Nb}_{14.3} \mathrm{~B}_{7}$, a value $E=0.1$ was found, indicating a high degree of collectivity. In contrast, for self-diffusion in crystalline Co one finds $E=0.7$. There, diffusion is by a vacancy mechanism that involves essentially single particle jumps with not too large displacements of the neighbors. The technique was applied to a supercooled melt of $\mathrm{Zr}_{46.7} \mathrm{Ti}_{8.3} \mathrm{Cu}_{7.5} \mathrm{Ni}_{10} \mathrm{Be}_{27.5}$, and again a very low isotope effect was observed [8].

Early molecular-dynamics simulation for hard disks and Lennard-Jones (LJ) systems found small isotope effects for mass ratios from 0.1 to 10 [9-11]. More recently, simulations of LJ systems found $E=0.2$ [12] and $E=0.1$ [13] for mass ratios of 2 and 4, respectively. Collective motion was also frequently observed in simulations of undercooling toward the glass transition $[14,15]$. This collectivity was attributed to stringlike motions [16-19] that resemble the relaxations in the amorphous material $[20,21]$.

The aim of the present paper is to present a systematic study of the diffusional isotope effect in a simple liquid as function of temperature and pressure. In order for the observed isotope effect to reflect the motion in the pure material, we study the effect of small mass changes, $10-40 \%$.

The calculations are done for a monatomic LJ system

$$
V(R)=4 \epsilon\left[(\sigma / R)^{12}-(\sigma / R)^{6}+A_{\mathrm{LJ}} R+B_{\mathrm{LJ}}\right] .
$$

The potential cutoff was set at $R_{c}=3 \sigma$. Similar to the shifted force potential [22], the parameters $A_{\mathrm{LJ}}=0.0095777$ and $B_{\mathrm{LJ}}=-0.00273595$ are introduced to ensure continuity of the potential and its first derivative at the cutoff. As usual, in the following, we will give all results in the appropriate units of energy $\epsilon$, length $\sigma$, and atomic mass $m$. The small shift is not expected to cause large differences to the phase diagram calculated for the pure LJ potential. For example, the melting temperature will be near the reported value, $T_{m} \approx 0.62[23]$. 


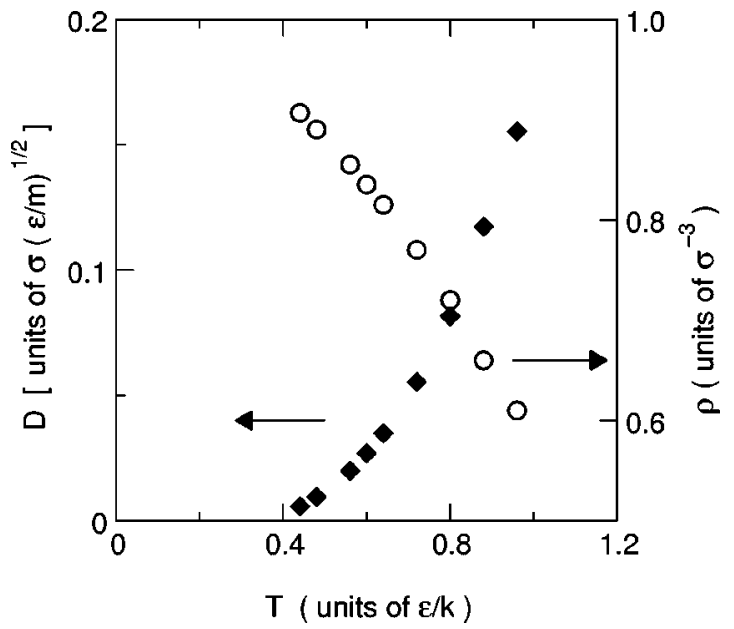

FIG. 1. Diffusion constants (full diamonds) and density (open circles) at zero pressure against temperature (all in reduced units).

The calculations were done with constant volume and periodic boundary conditions. The time step was $\Delta t=0.005$. Control runs with $\Delta t=0.0005$ showed no significant deviation. A heat bath was simulated by comparing the temperature averaged over 20 time steps with the nominal temperature. At each time step, $1 \%$ of the temperature difference was adjusted by random additions to the particle velocities. This procedure assured that existing correlations between the motion of atoms were only minimally affected. Again, control calculations were done with constant energy, and no significant effect was observed.

We prepared 11 samples, each of 5488 atoms, by heating amorphous samples to $T=0.88$. The samples were then equilibrated for 60000 time steps. From these samples, the ones at the other temperatures were gained by quenching at the appropriate volume followed by equilibration for 30000 time steps. Two series of calculations were done. For the first set the equilibrium densities $\rho(T)$ were determined and used to calculate the zero pressure diffusion constants as function of temperature in the range $0.44 \leqslant T \leqslant 0.96$. In a second calculation, the temperature was kept constant at $T=0.88$ and the density was varied, $0.46 \leqslant \rho \leqslant 0.855$. In all runs, pressure and energy were monitored to ensure stability of the configurations. The diffusion constant was calculated from the asymptotic slope of the atomic mean-square displacements.

Figure 1 shows the densities and diffusion constants for the zero-pressure configurations. The density agrees well with the ones given in Ref. [22] for a slightly different cutoff. The diffusion constant compares to the one given in [24]. It vanishes around $T=0.4$. An exact determination of this temperature is not possible due to the onset of crystallization during extended simulations.

To determine the isotope effect, the mass of two sets of 100 randomly chosen atoms was increased, respectively, diminished by $\delta m$. The mass change was usually taken as $\delta m=0.2 m$, with additional runs using $\delta m=0.1 m$ and $\delta m$ $=0.4 \mathrm{~m}$. This was done 54 times for each configuration giving in total a minimum of 594 configurations for each temperature and density. Figure 2 shows a typical result. To first order in $\delta m$, the curves for lower and higher masses are symmetric with respect to the $m=1$ curve. The total effect is very small, which necessitates the large statistics.

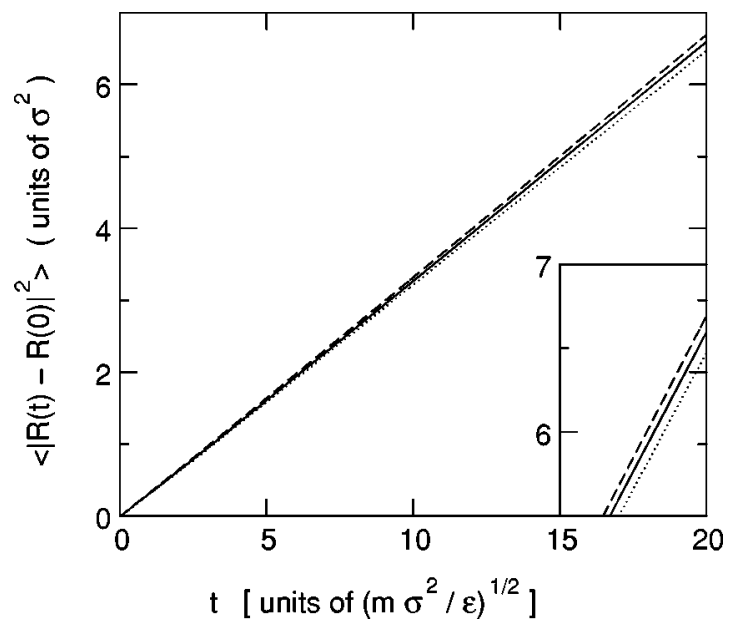

FIG. 2. Mean-square displacement as function of time for $T$ $=0.8$ and $\rho=0.72$. Solid line, $m=1$; dashed line, $m=0.8$; dotted line, $m=1.2$. The average is over 594 configurations and 200 time intervals each. The inset is an enlargement.

The mass dependence of the $D$ was then used to determine the isotope effect, Eq. (5). Taking the values for the zero-pressure samples in Fig. 3, one sees first that all the values are relatively low in the whole temperature range investigated and second, that $E$ drops more or less linearly upon cooling toward the glass transition temperature. This clearly shows a marked increase of motional collectivity upon cooling. Taking the approximate relation between $E$ and the number of particles moving collectively we find that the latter increases from $N_{D} \approx 4$ at $T=0.96$ to $N_{D} \approx 16$ at $T=0.48$. This high collectivity is in agreement with the experimental findings of Ehmler et al. [8] who found $E \approx 0.09$ for $\mathrm{Co}$ diffusion in supercooled liquid $\mathrm{Zr}_{46.7} \mathrm{Ti}_{8.3} \mathrm{Cu}_{7.5} \mathrm{Ni}_{10} \mathrm{Be}_{27.5}$. It is similar to the values found in the monatomic soft sphere glass at low temperatures [21] where collective jumps of approximately 20 atoms have been reported.

To check to what extent the drop of isotope effect is connected with the increase in density, the calculations were repeated at constant temperature $T=0.88$ for different densities $\rho$. Figure 4 (solid diamonds) shows an approximate pro-

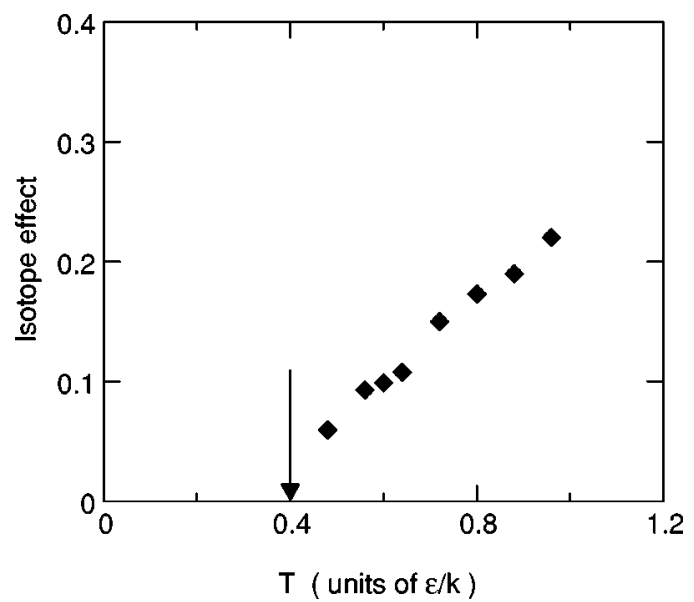

FIG. 3. Isotope effect as function of temperature at equilibrium density. 


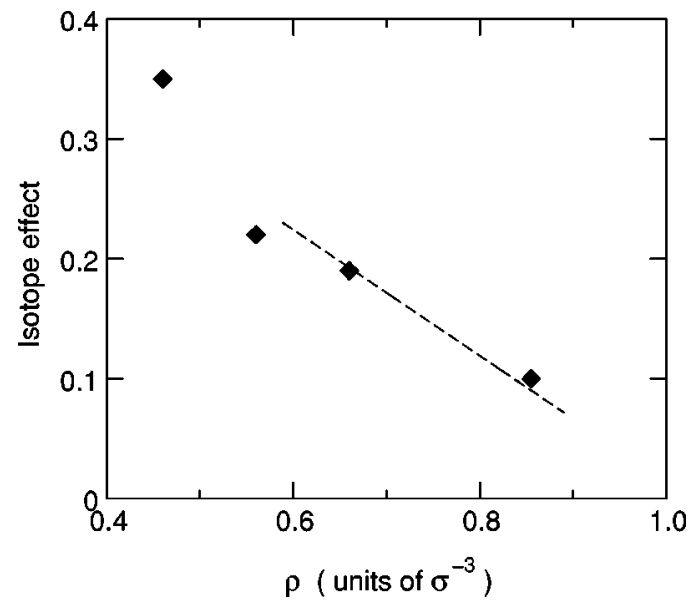

FIG. 4. Isotope effect as function of density for constant temperature $T=0.88$ (solid diamonds). The broken line is a least square fit to the data of Fig. 3 using the densities shown in Fig. 1.

portionality between the decrease of isotope effect and the increase of density. Moreover, the values at fixed temperature coincide within the limits of accuracy with those of the equilibrium samples (Fig. 3) at the same density but at a different temperature. This shows that the isotope effect is predominantly determined by the density. The diffusion constant itself depends strongly on both temperature and density.

Varying the mass increment $\delta m$ between 0.1 and 0.4 , we found no significant deviations from the simple square-root dependence of Eq. (4). Previous computer experiments on LJ liquids were done with much larger mass differences and different mass dependencies were deduced, e.g., $D\left(m_{1}\right) / D\left(m_{2}\right)=\left(m_{2} / m_{1}\right)^{-\mu}$, where $\mu<0.1$ [12]. From our results, we find that such a fit would require strongly temperature-dependent coefficients.

In experiments on metallic glasses, significantly larger isotope effects have been observed in rapidly quenched samples $[25] E \approx 0.5$. Upon aging, this value diminishes to $E \approx 0.1$ in the fully relaxed sample. In our simulation of a liquid LJ system, we observed a similar but less pronounced effect when using insufficiently equilibrated samples. We find for a very "young" sample at $T=0.88$ a value of $E$ $\approx 0.26$ that rapidly drops to $E \approx 0.19$ upon aging. The different temperature ranges and aging times prevent a quantitative comparison at this stage.

In the investigated temperature range, the increase in collectivity is not accompanied by a major increase of nonGaussianity, where Gaussianity is defined as [26]

$$
\alpha_{2}(t)=\frac{3\left\langle r^{4}(t)\right\rangle}{5\left\langle r^{2}(t)\right\rangle^{2}}-1,
$$

with $\left\langle r^{2}(t)\right\rangle$ and $\left\langle r^{4}(t)\right\rangle$ the mean-square displacement and the corresponding fourth-order term, respectively. A value $\alpha_{2}(t)=0$ indicates a Gaussian distribution of displacements as one would obtain from standard diffusion theory. At all temperatures, we find for all times $\alpha_{2}(t)<0.25$. This indicates that heterogeneity or atomic jumps over distances comparable to $\sigma$ play only a minor role. It is in contrast to results for binary LJ using constant volume conditions systems where $\alpha_{2}(t)$ approached maximal values of around 3 near the glass transition [27]. Below the glass transition, large non-Gaussianities have been observed in simulations of monatomic soft sphere glasses [21]. For the monatomic LJ system studied here, non-Gaussianity is found to increase at temperatures closer to $T_{g}$ than used in the study of the isotope effect. A long time study is precluded at these temperatures by the onset of crystallization.

In summary, we studied the isotope effect of diffusion in a simple Lennard-Jones liquid at equilibrium density as function of temperature and for constant temperature as function of density. The isotope effect drops strongly upon cooling toward the melting and glass transition temperatures and is strongly dependent on the densities. This indicates a strong increase in cooperativity of the atomic motion. The calculations were done for small mass differences and the results are in agreement with recent experiments. Incompletely equilibrated samples show an increased isotope effect-a less cooperative motion. Changing the mass ratio, no deviation from the simple description in terms of effective masses was found.
[1] H. J. V. Tyrrell and K. R. Harris, Diffusion in Liquids (Butterworth, London, 1984).

[2] U. Balucani and M. Zoppi, Dynamics of the Liquid State (Clarendon Press, Oxford, 1994).

[3] U. Mohanty, Adv. Chem. Phys. 89, 89 (1995).

[4] Slow Dynamics in Complex Systems, edited by M. Tokuyama and I. Oppenheim, AIP Conf. Proc. No. 469 (AIP, Woodbury, NY, 1999).

[5] R. W. Keyes, J. Chem. Phys. 29, 467 (1958).

[6] G. J. Krüger, W. Müller-Warmuth, and A. Klemm, Z. Naturforsch. 26a, 94 (1971).

[7] F. Faupel, P. W. Hüppe, and K. Rätzke, Phys. Rev. Lett. 65, 1219 (1990).

[8] H. Ehmler et al., Phys. Rev. Lett. 80, 4919 (1998).

[9] P. T. Herman and B. J. Alder, J. Chem. Phys. 56, 987 (1972).

[10] B. J. Alder, W. E. Alley, and J. H. Dymond, J. Chem. Phys. 61, 1415 (1974).
[11] I. Ebbsjö, P. Schofield, K. Sköld, and I. Waller, J. Phys. C 7, 3891 (1974).

[12] R. J. Bearman and D. L. Jolly, Mol. Phys. 44, 665 (1981).

[13] M. J. Nuevo, J. J. Morales, and D. M. Heyes, Phys. Rev. E 51, 2026 (1995).

[14] H. Miyagawa, Y. Hiwatari, B. Bernu, and J. Hansen, J. Chem. Phys. 88, 3879 (1988).

[15] G. Wahnström, Phys. Rev. A 44, 3752 (1991).

[16] H. R. Schober, C. Gaukel, and C. Oligschleger, Defect Diffus. Forum 143-147, 723 (1997).

[17] H. R. Schober, C. Gaukel, and C. Oligschleger, Prog. Theor. Phys. Suppl. 126, 67 (1997).

[18] C. Donati et al., Phys. Rev. Lett. 80, 2338 (1998).

[19] T. B. Schrøder and J. C. Dyre, J. Non-Cryst. Solids 235-237, 331 (1998).

[20] H. R. Schober, C. Oligschleger, and B. B. Laird, J. Non-Cryst. Solids 156, 965 (1993). 
[21] C. Oligschleger and H. R. Schober, Phys. Rev. B 59, 811 (1999).

[22] J. J. Nicolas, K. E. Gubbins, W. B. Streett, and D. J. Tildesley, Mol. Phys. 37, 1429 (1979).

[23] J. Q. Broughton, G. H. Gilmer, and K. A. Jackson, Phys. Rev. Lett. 49, 1496 (1982).
[24] M. J. Nuevo, J. J. Morales, and D. M. Heyes, Phys. Rev. E 55, 4217 (1997).

[25] K. Rätzke, P. W. Hüppe, and F. Faupel, Phys. Rev. Lett. 68, 2347 (1992).

[26] A. Rahman, Phys. Rev. 136, A405 (1964).

[27] W. Kob and H. C. Andersen, Phys. Rev. E 51, 4626 (1995). 